Supplement of The Cryosphere Discuss., 8, 2425-2463, 2014

http://www.the-cryosphere-discuss.net/tcd-8-2425-2014/

doi:10.5194/tcd-8-2425-2014-supplement

(C) Author(s) 2014. CC Attribution 3.0 License.

(c) (1)

Supplement of

\title{
Orientation dependent glacial changes at the Tibetan Plateau derived from
} 2003-2009 ICESat laser altimetry

\section{H. Phan et al.}

Correspondence to: V. H. Phan (phanhienvu@gmail.com) 
SUPPLEMENT

Table S1: Rates of glacial vertical changes on the Tibetan Plateau between 2003 and 2009

\begin{tabular}{|c|c|c|c|c|c|c|c|c|c|c|c|c|c|c|c|c|c|c|c|}
\hline & & Gla & ial area & & & $\mathrm{s} \leq 15$ & leg and & $R \leq 15 n$ & & & $S \leq 20$ & deg and & $\mathrm{R} \leq 15 \mathrm{~m}$ & & & $S \leq 25$ & eg and & $R \leq 15 \mathrm{~m}$ & \\
\hline No. & Lat. & Lon. & Orient. & Basin & $\mathrm{N}$ & $\begin{array}{c}V \\
(\mathrm{~m} / \mathrm{yr})\end{array}$ & $\begin{array}{c}\sigma_{\mathrm{vv}} \\
(\mathrm{m} / \mathrm{yr})\end{array}$ & $\begin{array}{c}\text { RMSE } \\
(\mathrm{m})\end{array}$ & $\kappa$ & $\mathrm{N}$ & $\begin{array}{c}V \\
(\mathrm{~m} / \mathrm{yr})\end{array}$ & $\begin{array}{c}\sigma_{\mathrm{vv}} \\
(\mathrm{m} / \mathrm{yr})\end{array}$ & $\begin{array}{c}\text { RMSE } \\
(\mathrm{m})\end{array}$ & $\kappa$ & $\mathrm{N}$ & $\begin{array}{c}V \\
(\mathrm{~m} / \mathrm{yr})\end{array}$ & $\begin{array}{c}\sigma_{\mathrm{vv}} \\
(\mathrm{m} / \mathrm{yr})\end{array}$ & $\begin{array}{c}\text { RMSE } \\
(\mathrm{m})\end{array}$ & $\kappa$ \\
\hline 1 & 28.2483 & 90.5435 & $\mathrm{~N}$ & Brahmaputra & & & & & & 71 & -0.14 & 0.40 & 7.13 & 0.0080 & 111 & 0.70 & 0.40 & 6.12 & 0.0124 \\
\hline 2 & 30.3370 & 93.6944 & $\mathrm{~N}$ & Brahmaputra & & & & & & 75 & 2.38 & 0.38 & 6.37 & 0.0034 & 98 & 2.30 & 0.56 & 8.08 & 0.0045 \\
\hline 3 & 30.3640 & 93.9454 & $\mathrm{~N}$ & Brahmaputra & & & & & & & & & & & 63 & 0.32 & 1.00 & 7.83 & 0.0038 \\
\hline 4 & 28.0525 & 90.5693 & $\mathrm{NE}$ & Brahmaputra & & & & & & 63 & -0.48 & 0.61 & 3.76 & 0.0094 & 82 & -0.67 & 0.59 & 7.12 & 0.0122 \\
\hline 5 & 29.2107 & 97.3078 & $\mathrm{NE}$ & Brahmaputra & & & & & & 73 & -1.06 & 0.73 & 7.91 & 0.0087 & 87 & -3.04 & 0.65 & 5.82 & 0.0104 \\
\hline 6 & 29.3733 & 96.6197 & $\mathrm{NE}$ & Brahmaputra & 128 & -0.26 & 0.42 & 4.18 & 0.0024 & 181 & -1.56 & 0.49 & 6.12 & 0.0033 & 187 & -1.56 & 0.48 & 6.39 & 0.0034 \\
\hline 7 & 29.7669 & 82.8037 & $\mathrm{NE}$ & Brahmaputra & 248 & -1.04 & 0.31 & 2.51 & 0.0138 & 262 & -1.09 & 0.33 & 2.33 & 0.0146 & 273 & -1.10 & 0.40 & 2.38 & 0.0152 \\
\hline 8 & 27.9793 & 92.7260 & $E$ & Brahmaputra & & & & & & & & & & & 86 & 0.26 & 0.76 & 7.31 & 0.0040 \\
\hline 9 & 29.6202 & 96.0862 & $E$ & Brahmaputra & & & & & & 76 & -2.03 & 0.73 & 0.32 & 0.0043 & 102 & -2.68 & 0.81 & 1.82 & 0.0057 \\
\hline 10 & 30.3395 & 93.1502 & $E$ & Brahmaputra & & & & & & & & & & & 75 & 0.94 & 0.65 & 5.96 & 0.0181 \\
\hline 11 & 30.3670 & 93.8065 & $\bar{E}$ & \begin{tabular}{|l|} 
Brahmaputra \\
\end{tabular} & & & & & & & & & & & 52 & 0.39 & 0.93 & 6.06 & 0.0037 \\
\hline 12 & 30.6042 & 95.2404 & $\mathrm{E}$ & Brahmaputra & & & & & & & & & & & 62 & -0.44 & 0.70 & 3.27 & 0.0104 \\
\hline 13 & 30.1126 & 90.2632 & $\mathrm{SE}$ & Brahmaputra & & & & & & & & & & & 54 & 0.30 & 0.86 & 5.95 & 0.0185 \\
\hline 14 & 30.1705 & 90.2812 & $\mathrm{SE}$ & Brahmaputra & & & & & & & & & & & 58 & -2.86 & 0.50 & 6.67 & 0.0183 \\
\hline 15 & 30.4785 & 94.6782 & $\mathrm{SE}$ & Brahmaputra & 369 & -0.81 & 0.28 & 4.83 & 0.0030 & 444 & -0.97 & 0.32 & 4.44 & 0.0036 & 557 & -1.24 & 0.44 & 3.94 & 0.0045 \\
\hline 16 & 28.1838 & 90.5439 & $\mathrm{~s}$ & Brahmaputra & 195 & -0.72 & 0.36 & 6.80 & 0.0142 & 261 & -0.09 & 0.39 & 8.68 & 0.0190 & 309 & 0.12 & 0.43 & 9.15 & 0.0226 \\
\hline 17 & 28.0152 & 88.3758 & $\mathrm{~N}$ & Ganges & & & & & & 61 & -0.95 & 0.81 & 2.63 & 0.0239 & 88 & -2.11 & 0.69 & 3.15 & 0.0345 \\
\hline 18 & 28.0554 & 86.8359 & $\mathrm{~N}$ & Ganges & 189 & -1.77 & 0.32 & 4.77 & 0.0091 & 239 & -2.09 & 0.36 & 7.61 & 0.0115 & 328 & -1.23 & 0.42 & 9.64 & 0.0157 \\
\hline 19 & 28.3360 & 86.3018 & $\mathrm{~N}$ & Ganges & 84 & -0.06 & 0.20 & 2.12 & 0.0117 & 93 & 0.12 & 0.25 & 4.64 & 0.0129 & 103 & 0.01 & 0.25 & 3.09 & 0.0143 \\
\hline 20 & 30.4692 & 81.3096 & $\mathrm{~N}$ & Ganges & 94 & -0.71 & 0.52 & 3.63 & 0.0212 & 99 & -0.74 & 0.54 & 3.40 & 0.0223 & 108 & -0.53 & 0.46 & 3.25 & 0.0243 \\
\hline 21 & 31.0027 & 79.2772 & $\mathrm{~N}$ & Ganges & 207 & -1.61 & 0.20 & 4.95 & 0.0139 & 236 & -1.39 & 0.23 & 4.87 & 0.0158 & 250 & -1.37 & 0.31 & 5.00 & 0.0168 \\
\hline 22 & 28.6863 & 85.4509 & $\mathrm{NE}$ & Ganges & & & & & & & & & & & 70 & -0.39 & 0.36 & 6.90 & 0.0117 \\
\hline 23 & 28.1540 & 86.7890 & $E$ & Ganges & & & & & & 74 & -0.17 & 0.52 & 2.97 & 0.0110 & 99 & -0.36 & 0.52 & 4.28 & 0.0147 \\
\hline 24 & \begin{tabular}{|l|}
28.2613 \\
\end{tabular} & 86.2958 & $\mathrm{~s}$ & \begin{tabular}{|l|} 
Ganges \\
\end{tabular} & 261 & -2.01 & 0.30 & 3.91 & 0.0228 & 323 & -1.83 & 0.37 & 3.40 & 0.0283 & 361 & -1.82 & 0.40 & 3.41 & 0.0316 \\
\hline 25 & 30.4152 & 81.3064 & $\mathrm{~s}$ & Ganges & 75 & -0.38 & 0.68 & 5.91 & 0.0260 & 80 & -0.90 & 0.69 & 5.83 & 0.0278 & 82 & -0.94 & 0.70 & 5.81 & 0.0285 \\
\hline 26 & 31.1669 & 79.3033 & NW & Ganges & & & & & & & & & & & 72 & 1.19 & 0.69 & 9.28 & 0.0418 \\
\hline 27 & 34.1788 & 78.9947 & $\mathrm{~N}$ & Indus & & & & & & 77 & 0.74 & 0.35 & 2.12 & 0.0280 & 109 & 1.43 & 0.40 & 3.26 & 0.0396 \\
\hline 28 & 32.7876 & 81.0514 & $E$ & Indus & 60 & -0.69 & 0.51 & 3.01 & 0.0111 & 128 & 0.65 & 0.71 & 4.86 & 0.0237 & 169 & 0.13 & 0.72 & 5.11 & 0.0313 \\
\hline 29 & 34.0527 & 79.7882 & $E$ & Indus & 178 & -0.05 & 0.19 & 1.59 & 0.0761 & 185 & -0.07 & 0.20 & 1.51 & 0.0791 & 191 & 0.03 & 0.20 & 2.77 & 0.0817 \\
\hline 30 & 34.2232 & 79.8126 & $\mathrm{~s}$ & Indus & 106 & -0.11 & 0.01 & 1.67 & 0.1105 & 106 & -0.11 & 0.01 & 1.67 & 0.1105 & 106 & -0.11 & 0.01 & 1.67 & 0.1105 \\
\hline 31 & 34.0245 & 79.7631 & SW & Indus & 63 & -0.85 & \begin{tabular}{l|l}
0.39 \\
\end{tabular} & 2.20 & 0.0186 & 79 & \begin{tabular}{|c|}
-1.38 \\
\end{tabular} & 0.43 & 2.73 & 0.0234 & 87 & -1.47 & 0.47 & 3.22 & 0.0257 \\
\hline 32 & 31.1226 & 83.4559 & $\mathrm{~N}$ & Inner plateau & 58 & -1.31 & 0.50 & 2.55 & 0.0117 & 68 & -0.71 & 0.47 & 3.61 & 0.0138 & 74 & -0.69 & 0.48 & 2.77 & 0.0150 \\
\hline 33 & 33.9313 & 90.4148 & $\mathrm{~N}$ & Inner plateau & 49 & 0.38 & 0.38 & 2.69 & 0.0154 & 63 & 0.48 & 0.46 & 3.17 & 0.0198 & 68 & 0.62 & 0.49 & 4.24 & 0.0213 \\
\hline 34 & 34.3265 & 81.9460 & $\mathrm{~N}$ & Inner plateau & 150 & 0.25 & 0.38 & 3.07 & 0.0355 & 168 & 0.21 & 0.47 & 2.25 & 0.0398 & 193 & 0.55 & 0.49 & 3.64 & 0.0457 \\
\hline 35 & 34.5128 & 80.7636 & $\mathrm{~N}$ & Inner plateau & & & & & & & & & & & 79 & -1.77 & 0.89 & 6.89 & 0.0428 \\
\hline 36 & 35.6960 & 85.6129 & $\mathrm{~N}$ & Inner plateau & 220 & -0.34 & 0.24 & 0.98 & 0167 & 257 & -0.04 & 0.24 & 2.85 & 0.0195 & 284 & 0.09 & 0.25 & 5.08 & 0.0216 \\
\hline 37 & $\begin{array}{l}36.0994 \\
\end{array}$ & 90.9355 & $\mathrm{~N}$ & Inner plateau & 444 & -0.41 & 0.20 & 2.62 & 0.0090 & 494 & -0.55 & 0.22 & 2.88 & 0.0100 & 531 & -0.48 & 0.25 & 2.73 & 0.0107 \\
\hline 38 & 30.9295 & 82.9716 & $\mathrm{NE}$ & Inner plateau & 48 & -1.38 & 1.15 & 3.90 & 0.0064 & 74 & -1.13 & 0.82 & 4.28 & 0.0099 & 97 & -1.59 & 0.69 & 5.86 & 0.0130 \\
\hline 39 & |33.6898 & 82.4899 & $\mathrm{NE}$ & Inner plateau & & & & & & & & & & & 68 & -2.43 & 1.35 & 4.81 & 0.0236 \\
\hline 40 & 34.3768 & 79.8450 & $\mathrm{NE}$ & Inner plateau & 138 & 0.16 & 0.39 & 3.38 & 0.0161 & 210 & -0.03 & 0.46 & 1.85 & 0.0246 & 245 & 0.17 & 0.51 & 2.06 & 0.0287 \\
\hline 41 & 30.6121 & 86.4643 & $E$ & Inner plateau & & & & & & 46 & -4.38 & 0.98 & 5.99 & 0.0042 & 60 & -1.94 & 0.99 & 5.89 & 0.0054 \\
\hline 42 & 30.9359 & 83.4939 & $E$ & Inner plateau & 55 & 0.43 & 0.78 & 6.44 & 0.0181 & 83 & 1.63 & 0.58 & 9.21 & 0.0273 & 93 & 2.12 & 0.76 & 7.79 & 0.0306 \\
\hline 43 & 33.3058 & 91.3293 & $\mathrm{SE}$ & Inner plateau & & & & & & & & & & & 62 & -3.15 & 1.18 & 4.60 & 0.0321 \\
\hline 44 & 33.4696 & 86.7921 & $\mathrm{SE}$ & Inner plateau & & & & & & & & & & & 53 & -1.37 & 0.81 & 5.38 & 0.0226 \\
\hline 45 & 35.4881 & 82.1995 & $\mathrm{SE}$ & Inner plateau & 58 & 0.91 & 0.83 & 2.54 & 0.0140 & 100 & 1.01 & 0.87 & 5.04 & 0.0242 & 132 & 2.45 & 0.67 & 7.54 & 0.0320 \\
\hline 46 & 35.5528 & 89.6168 & SE & Inner plateau & 161 & 0.12 & 0.40 & 3.03 & 0.0176 & 192 & -0.06 & 0.61 & 4.45 & 0.0210 & 212 & -0.48 & 0.69 & 4.40 & 0.0232 \\
\hline 47 & 35.8736 & 91.4318 & $\mathrm{SE}$ & Inner plateau & 46 & 1.01 & 0.45 & 3.49 & 0.0184 & 85 & \begin{tabular}{l|l}
0.18 \\
\end{tabular} & 0.61 & 3.67 & 0.0340 & 101 & -0.13 & 0.59 & 4.28 & 0.0404 \\
\hline 48 & 33.9128 & 90.6589 & $\mathrm{~s}$ & Inner plateau & 57 & -0.59 & 0.20 & 3.16 & 0.0269 & 92 & -0.47 & 0.20 & 3.92 & 0.0433 & 105 & -0.05 & 0.20 & 4.74 & 0.0495 \\
\hline 49 & $\begin{array}{l}34.2879 \\
\end{array}$ & 81.9455 & $\mathrm{~s}$ & Inner plateau & 63 & 1.54 & 0.49 & 2.56 & 0.0188 & 106 & 1.23 & 0.49 & 2.76 & 0.0316 & 116 & 1.23 & 0.49 & 2.83 & 0.0346 \\
\hline 50 & 35.2841 & 80.6850 & $\mathrm{~s}$ & Inner plateau & 815 & -1.41 & \begin{tabular}{l|l|}
0.27 \\
\end{tabular} & 4.65 & 0.0078 & 998 & -1.02 & 0.29 & 4.19 & 0.0096 & 1150 & -0.84 & 0.30 & 4.08 & 0.0111 \\
\hline \begin{tabular}{l|l}
51 \\
\end{tabular} & 35.4700 & 82.1430 & $\mathrm{~s}$ & Inner plateau & 67 & 1.18 & 1.13 & 2.67 & 0.0198 & 92 & -1.50 & 0.79 & 4.41 & 0.0271 & 118 & -1.30 & 0.66 & 5.56 & 0.0348 \\
\hline 52 & 35.6549 & 85.6200 & $\mathrm{~s}$ & Inner plateau & 55 & 2.01 & 0.63 & 3.82 & 0.0046 & 118 & 1.82 & 0.48 & 5.08 & 0.0100 & 159 & 1.69 & 0.54 & 5.34 & 0.0134 \\
\hline 53 & 36.7727 & 84.9026 & $\mathrm{~s}$ & Inner plateau & & & & & & 59 & -0.13 & 0.56 & 2.89 & 0.0246 & 80 & 0.13 & 0.62 & 5.10 & 0.0333 \\
\hline 54 & 34.3105 & 85.8384 & SW & Inner plateau & 284 & 0.60 & 0.17 & 2.27 & 0.0358 & 310 & 0.81 & 0.20 & 2.66 & 0.0391 & 322 & 0.76 & 0.20 & 3.37 & 0.0406 \\
\hline 55 & 35.3008 & 81.4300 & SW & Inner plateau & 574 & -0.26 & 0.19 & 1.71 & 0.0270 & 635 & -0.29 & 0.20 & 1.73 & 0.0298 & 661 & -0.31 & 0.20 & 1.93 & 0.0311 \\
\hline 56 & 31.0217 & 83.4683 & $\mathrm{~W}$ & Inner plateau & 139 & -0.14 & 0.28 & 3.80 & 0.0113 & 160 & -0.46 & 0.36 & 3.56 & 0.0130 & 189 & -0.59 & 0.46 & 4.31 & 0.0153 \\
\hline 57 & 34.7324 & 82.3218 & $\mathrm{w}$ & Inner plateau & 245 & -0.70 & 0.30 & 1.71 & 0.0165 & 294 & \begin{tabular}{l|l|}
-0.39 \\
\end{tabular} & 0.34 & 2.72 & 0.0198 & 311 & -0.50 & 0.39 & 3.07 & 0.0210 \\
\hline 58 & 33.4831 & 86.7867 & NW & Inner plateau & & & & & & & & & & & 51 & 1.17 & 0.99 & 2.79 & 0.0318 \\
\hline 59 & 34.3519 & 79.9857 & NW & $\operatorname{lnner} \mathrm{p}$ & 226 & 0.21 & 0.22 & 2.58 & 0.0319 & 242 & 0.11 & 0.25 & 3.69 & 0.0342 & 266 & 0.31 & 0.25 & 8.13 & 0.0375 \\
\hline 60 & 34.3766 & 85.8347 & NW & Inner plateau & & & & & & & & & & & 74 & -0.57 & 0.72 & 3.62 & 0.0169 \\
\hline 61 & 33.5516 & 94.7536 & $\mathrm{~N}$ & Mekong & & & & & & & & & & & 74 & -1.01 & 0.48 & 6.18 & 0.0246 \\
\hline 62 & $\begin{array}{l}31.7083 \\
\end{array}$ & 95.8596 & $\mathrm{NE}$ & Mekong & & & & & & & & & & & 44 & -2.88 & 0.35 & 9.63 & 0.0245 \\
\hline 63 & 31.0089 & 93.8679 & $\mathrm{~N}$ & Salween & & & & & & & & & & & 51 & 0.35 & 0.71 & 7.51 & 0.0150 \\
\hline 64 & $\begin{array}{l}30.9253 \\
\end{array}$ & 93.8699 & $\mathrm{NE}$ & Salween & & & & & & & & & & & 53 & -0.99 & 0.78 & 3.56 & 0.0158 \\
\hline 65 & 30.6792 & 94.6483 & $E$ & Salween & & & & & & 58 & -0.78 & 0.81 & 4.18 & 0.0250 & 66 & -0.45 & 0.87 & 3.79 & 0.0285 \\
\hline 66 & 30.7695 & 94.6702 & $E$ & Salween & & & & & & & & & & & 39 & -0.54 & 0.46 & 3.49 & 0.0118 \\
\hline 67 & 35.2281 & 78.6456 & $\mathrm{~N}$ & Tarim Basin & & & & & & & & & & & 54 & -2.36 & 0.63 & 2.04 & 0.0134 \\
\hline 68 & 35.3878 & 81.3971 & $\mathrm{~N}$ & Tarim Basin & 5 & -0.18 & 0. & & 0.00 & 633 & -0.0 & 0.30 & 1.44 & 0.0082 & 656 & -0.08 & 0.31 & 1.57 & 0.0085 \\
\hline 69 & $\begin{array}{l}35.4884 \\
\end{array}$ & 77.6894 & $\mathrm{~N}$ & Tarim Basin & 162 & -0.31 & 0.20 & 3.94 & 0.0269 & 185 & -0.61 & 0.26 & 5.16 & 0.0307 & 199 & -0.79 & 0.33 & 5.54 & 0.0330 \\
\hline 70 & 35.5083 & 81.6237 & $\mathrm{~N}$ & Tarim Basin & 341 & 0.38 & 0.25 & 1.45 & 0.0240 & 380 & 0.58 & 0.28 & 1.79 & 0.0267 & 404 & 0.43 & 0.31 & 2.02 & 0.0284 \\
\hline 71 & 35.5157 & 82.1624 & $\mathrm{~N}$ & Tarim Basin & & & & & & 77 & -1.02 & 0.43 & 5.07 & 0.0107 & 129 & 2.32 & 0.56 & 7.49 & 0.0179 \\
\hline 72 & 35.5234 & 80.7134 & $\mathrm{~N}$ & Tarim Basin & 1120 & 0.50 & 0.24 & 3.62 & 0.0076 & 1320 & 0.69 & 0.30 & 3.38 & 0.0090 & 1497 & 0.69 & 0.34 & 3.01 & 0.0102 \\
\hline 73 & 35.6376 & 80.0381 & $\mathrm{~N}$ & Tarim Basin & 308 & 0.54 & 0.20 & 2.16 & 0.0312 & 343 & 0.60 & 0.33 & 2.08 & 0.0348 & 373 & 0.89 & 0.41 & 2.90 & 0.0378 \\
\hline 74 & 35.6398 & 77.1217 & $\mathrm{~N}$ & Tarim Basin & 77 & -0.91 & 0.27 & 2.33 & 0.0011 & 116 & 0.22 & 0.33 & 2.23 & 0.0016 & 190 & -0.33 & 0.34 & 3.98 & 0.0027 \\
\hline 75 & 35.7032 & 77.6240 & $\mathrm{~N}$ & Tarim Basin & & & & & & & & & & & 76 & -0.49 & 0.53 & 2.78 & 0.0157 \\
\hline 76 & $\begin{array}{l}35.7379 \\
\end{array}$ & 76.8019 & $\mathrm{~N}$ & Tarim Basin & & & & & & 59 & -0.78 & 0.40 & 7.49 & 0.0012 & 73 & -0.98 & 0.50 & 9.06 & 0.0015 \\
\hline 77 & 35.7461 & 78.7394 & $\mathrm{~N}$ & Tarim Basin & 70 & 0.45 & 0.42 & 3.12 & 0.0147 & 116 & 2.10 & 0.46 & 7.16 & 0.0244 & 145 & 1.97 & 0.64 & 4.31 & 0.0305 \\
\hline
\end{tabular}




\begin{tabular}{|c|c|c|c|c|c|c|c|c|c|c|c|c|c|c|c|c|c|c|c|}
\hline 78 & 35.7896 & 93.3675 & $\mathrm{~N}$ & Tarim Basin & & & & & & & & & & & 76 & 1.23 & 0.68 & 10.17 & 0.0364 \\
\hline 79 & 35.8122 & 77.1481 & $\mathrm{~N}$ & Tarim Basin & & & & & & 47 & 0.19 & 0.57 & 3.16 & 0.0198 & 66 & 0.05 & 0.54 & 2.80 & 0.0278 \\
\hline 80 & 35.9110 & 91.4375 & $\mathrm{~N}$ & Tarim Basin & & & & & & & & & & & 59 & -3.40 & 0.86 & 2.49 & 0.0166 \\
\hline 81 & 35.9285 & 77.1800 & $\mathrm{~N}$ & Tarim Basin & 143 & 0.46 & 0.29 & 1.32 & 0.0060 & 187 & 0.25 & 0.36 & 5.32 & 0.0079 & 236 & -0.47 & 0.38 & 6.06 & 0.0099 \\
\hline 82 & 35.9310 & 80.8954 & $\mathrm{~N}$ & Tarim Basin & 45 & -0.74 & 0.57 & 2.05 & 0.0126 & 69 & -0.86 & 0.60 & 2.32 & 0.0194 & 88 & 0.09 & 0.54 & 2.98 & 0.0247 \\
\hline 83 & 36.0723 & 79.3273 & $\mathrm{~N}$ & Tarim Basin & & & & & & 56 & 1.25 & 0.51 & 3.09 & 0.0070 & 80 & 2.56 & 0.75 & 5.19 & 0.0101 \\
\hline 84 & 36.1386 & 75.9995 & $\mathrm{~N}$ & Tarim Basin & 470 & 0.92 & 0.27 & 5.71 & 0.0028 & 528 & 0.99 & 0.28 & 6.69 & 0.0031 & 576 & 1.15 & 0.29 & 6.30 & 0.0034 \\
\hline 85 & 36.4774 & 75.9075 & $\mathrm{~N}$ & Tarim Basin & & & & & & 52 & 0.00 & 0.63 & 2.98 & 0.0071 & 76 & 0.04 & 0.49 & 3.23 & 0.0103 \\
\hline 86 & 36.5223 & 77.4777 & $\mathrm{~N}$ & Tarim Basin & 93 & -0.12 & 0.49 & 2.87 & 0.0070 & 106 & -0.05 & 0.50 & 3.21 & 0.0079 & 129 & 0.30 & 0.49 & 3.36 & 0.0096 \\
\hline 87 & 36.6339 & 91.1211 & $\mathrm{~N}$ & Tarim Basin & & & & & & 127 & 1.38 & 0.53 & 5.72 & 0.0087 & 204 & 1.13 & 0.54 & 6.73 & 0.0140 \\
\hline 88 & 36.8127 & 84.8954 & $\mathrm{~N}$ & Tarim Basin & & & & & & 52 & 0.03 & 0.78 & 2.44 & 0.0164 & 70 & -1.55 & 0.77 & 5.35 & 0.0221 \\
\hline 89 & 36.9845 & 75.2616 & $\mathrm{~N}$ & Tarim Basin & & & & & & 62 & -0.42 & 0.66 & 2.46 & 0.0117 & 66 & -0.35 & 0.65 & 1.89 & 0.0125 \\
\hline 90 & 37.0809 & 75.2591 & $\mathrm{~N}$ & Tarim Basin & 49 & -0.44 & 0.69 & 1.44 & 0.0104 & 67 & -0.54 & 0.70 & 4.04 & 0.0143 & 103 & 0.46 & 1.00 & 5.47 & 0.0220 \\
\hline 91 & 37.3722 & 87.7941 & $\mathrm{~N}$ & Tarim Basin & 91 & 0.06 & 0.25 & 2.34 & 0.0477 & 92 & 0.07 & 0.25 & 2.34 & 0.0482 & 98 & 0.11 & 0.25 & 2.04 & 0.0514 \\
\hline 92 & 37.3798 & 87.7196 & $\mathrm{~N}$ & Tarim Basin & 49 & 0.63 & 0.29 & 1.05 & 0.0080 & 63 & 0.06 & 0.30 & 3.52 & 0.0102 & 70 & 0.17 & 0.31 & 2.51 & 0.0114 \\
\hline 93 & 37.5106 & 87.8260 & $\mathrm{~N}$ & Tarim Basin & & & & & & 107 & 0.13 & 0.39 & 4.83 & 0.0466 & 137 & 0.57 & 0.56 & 5.02 & 0.0597 \\
\hline 94 & 38.1895 & 96.3351 & $\mathrm{~N}$ & Tarim Basin & 138 & -0.57 & 0.31 & 3.04 & 0.0113 & 157 & -0.33 & 0.31 & 2.36 & 0.0129 & 181 & -0.68 & 0.35 & 3.12 & 0.0149 \\
\hline 95 & 39.2061 & 73.5100 & $\mathrm{~N}$ & Tarim Basin & 85 & -1.03 & 0.50 & 4.29 & 0.0371 & 111 & -0.47 & 0.50 & 4.91 & 0.0484 & 112 & -0.47 & 0.50 & 5.00 & 0.0489 \\
\hline 96 & 36.0925 & 80.1294 & $\mathrm{NE}$ & Tarim Basin & & & & & & & & & & & 52 & 0.49 & 0.42 & 6.64 & 0.0120 \\
\hline 97 & 36.4999 & 91.3473 & $\mathrm{NE}$ & Tarim Basin & & & & & & & & & & & 85 & -1.10 & 0.85 & 4.43 & 0.0229 \\
\hline 98 & 36.5810 & 77.2859 & $\mathrm{NE}$ & Tarim Basin & & & & & & & & & & & 53 & -0.25 & 0.52 & 5.22 & 0.0143 \\
\hline 99 & 36.8056 & 76.6516 & $\mathrm{NE}$ & Tarim Basin & & & & & & 73 & 0.75 & 0.34 & 3.43 & 0.0244 & 94 & 1.73 & 0.47 & 3.23 & 0.0314 \\
\hline 100 & 37.7371 & 101.4823 & $\mathrm{NE}$ & Tarim Basin & & & & & & 39 & 1.69 & 0.57 & 5.63 & 0.0173 & 57 & 1.12 & 0.54 & 5.25 & 0.0253 \\
\hline 101 & 38.2362 & 89.2855 & $\mathrm{NE}$ & Tarim Basin & & & & & & & & & & & 60 & 0.90 & 0.52 & 6.36 & 0.0304 \\
\hline 102 & 38.2380 & 95.9301 & $\mathrm{NE}$ & Tarim Basin & & & & & & 65 & 0.81 & 0.70 & 6.49 & 0.0300 & 80 & 0.05 & 0.64 & 6.64 & 0.0370 \\
\hline 103 & 35.9769 & 78.9192 & $E$ & Tarim Basin & & & & & & 49 & 1.10 & 0.61 & 7.50 & 0.0136 & 77 & 1.83 & 0.66 & 6.87 & 0.0214 \\
\hline 104 & 36.7870 & 75.8619 & $\mathrm{E}$ & Tarim Basin & & & & & & 96 & 1.09 & 0.50 & 4.84 & 0.0163 & 135 & 1.17 & 0.54 & 5.37 & 0.0229 \\
\hline 105 & 37.6474 & 88.2015 & $\mathrm{E}$ & Tarim Basin & & & & & & 52 & 0.21 & 0.44 & 3.22 & 0.0403 & 60 & -0.13 & 0.60 & 4.74 & 0.0466 \\
\hline 106 & 35.4097 & 81.6117 & SE & Tarim Basin & 301 & -0.42 & 0.41 & 2.98 & 0.0296 & 338 & -0.44 & 0.44 & 3.46 & 0.0332 & 360 & -0.51 & 0.51 & 4.05 & 0.0354 \\
\hline 107 & 38.8890 & 73.9813 & $\mathrm{SE}$ & Tarim Basin & & & & & & & & & & & 46 & 0.27 & 0.49 & 2.65 & 0.0430 \\
\hline 108 & 39.3418 & 73.5386 & SE & Tarim Basin & 99 & 1.97 & 0.60 & 2.08 & 0.0140 & 135 & 1.34 & 0.84 & 2.64 & 0.0191 & 164 & -0.85 & 0.86 & 4.64 & 0.0232 \\
\hline 109 & 36.0236 & 90.9623 & $S$ & Tarim Basin & 381 & -0.66 & 0.36 & 3.07 & 0.0072 & 428 & -0.80 & 0.38 & 7.03 & 0.0081 & 487 & -1.12 & 0.47 & 7.59 & 0.0092 \\
\hline 110 & 38.8333 & 74.9679 & SW & Tarim Basin & 142 & 0.06 & 0.38 & 2.27 & 0.0259 & 145 & 0.09 & 0.39 & 2.03 & 0.0265 & 146 & -0.03 & 0.39 & 2.23 & 0.0267 \\
\hline 111 & 35.7742 & 77.1298 & W & Tarim Basin & 65 & 0.98 & 0.34 & 2.12 & 0.0046 & 93 & 0.06 & 0.57 & 4.74 & 0.0066 & 138 & 0.11 & 0.68 & 7.20 & 0.0098 \\
\hline 112 & 36.3990 & 78.8542 & $W$ & Tarim Basin & 88 & 0.14 & 0.25 & 2.72 & 0.0287 & 116 & -0.07 & 0.44 & 3.27 & 0.0378 & 139 & -0.23 & 0.39 & 3.49 & 0.0453 \\
\hline 113 & 38.2403 & 75.0779 & $\mathrm{~W}$ & Tarim Basin & & & & & & & & & & & 107 & -1.13 & 0.96 & 5.50 & 0.0048 \\
\hline 114 & 39.0528 & 74.9451 & $W$ & Tarim Basin & & & & & & & & & & & 43 & 2.05 & 1.42 & 5.01 & 0.0038 \\
\hline 115 & 36.6042 & 77.4907 & NW & Tarim Basin & 96 & 1.24 & 0.27 & 2.64 & 0.0199 & 129 & 0.77 & 0.48 & 3.70 & 0.0267 & 156 & -0.58 & 0.50 & 5.86 & 0.0323 \\
\hline 116 & 38.7759 & 73.9556 & $\mathrm{NW}$ & Tarim Basin & & & & & & 66 & -1.96 & 0.51 & 2.38 & 0.0403 & 105 & -0.41 & 0.94 & 7.20 & 0.0641 \\
\hline 117 & 38.9077 & 73.9818 & NW & Tarim Basin & & & & & & & & & & & 58 & 0.56 & 0.62 & 6.39 & 0.0465 \\
\hline 118 & 33.4012 & 91.3401 & $\mathrm{~N}$ & Yangtze & 108 & -0.61 & 0.54 & 3.41 & 0.0431 & 135 & -1.67 & 0.48 & 3.91 & 0.0538 & 169 & -0.96 & 0.48 & 3.95 & 0.0674 \\
\hline 119 & 33.9536 & 90.6697 & $\mathrm{~N}$ & Yangtze & 324 & -0.48 & 0.29 & 3.19 & 0.0323 & 342 & -0.60 & 0.30 & 3.23 & 0.0341 & 354 & -0.61 & 0.32 & 3.55 & 0.0353 \\
\hline 120 & 29.6724 & 101.9318 & $E$ & Yangtze & 70 & -0.39 & 0.59 & 4.23 & 0.0017 & 101 & -0.45 & 0.48 & 5.14 & 0.0025 & 121 & -0.67 & 0.54 & 7.19 & 0.0030 \\
\hline 121 & 33.1281 & 92.1241 & $E$ & Yangtze & 98 & -1.19 & 0.53 & 2.25 & 0.0272 & 131 & -1.37 & 0.55 & 2.92 & 0.0363 & 158 & -0.59 & 0.56 & 4.30 & 0.0438 \\
\hline 122 & 33.0758 & 92.1159 & $S$ & Yangtze & 42 & -0.98 & 0.77 & 3.64 & 0.0189 & 67 & -1.63 & 0.49 & 4.54 & 0.0301 & 83 & -0.46 & 0.59 & 6.37 & 0.0373 \\
\hline
\end{tabular}

